\title{
On Localization Properties of Damage Models
}

\author{
Milan Jirásek ${ }^{a}$ and Martin Horák ${ }^{b}$ \\ Department of Mechanics, Faculty of Civil Engineering \\ Czech Technical University in Prague, 16629 Prague, Czech Republic \\ amilan.jirasek@fsv.cvut.cz, ${ }^{\mathrm{b}}$ nitramkaroh@seznam.cz
}

Keywords: damage, strain localization, softening, bifurcation, weak discontinuity.

\begin{abstract}
Continuum damage mechanics provides an appropriate modeling framework for materials weakened by evolving defects such as cracks or voids. However, local damage models fail to provide an objective description of the material behavior after the loss of ellipticity, which can be detected by classical methods of localization analysis based on the acoustic tensor. This paper presents a careful analysis of localization properties of a class of isotropic damage models with one damage variable and with different definitions of the damage-driving equivalent strain. The necessary conditions for an incipient weak discontinuity under plane stress are compared, discussed, and illustrated by numerical examples.
\end{abstract}

\section{Introduction}

Realistic description of the mechanical behavior of quasibrittle materials such as concrete requires constitutive laws with softening. The physical origin of softening is in the propagation and coalescence of defects such as voids or cracks. It is well known that softening may lead to localization of inelastic strain into narrow process zones. For traditional models formulated within the classical framework of continuum mechanics, such zones have an arbitrarily small thickness, and failure can occur at arbitrarily small energy dissipation, which is not realistic. The mathematical model becomes ill-posed and the numerical solutions suffer by pathological sensitivity to the discretization parameter, e.g. to the size of finite elements. It is therefore important to clearly understand the conditions under which localization may occur, and to limit the application of traditional continuum damage mechanics to the range of material states that do not allow for localization of damage into arbitrarily thin bands. Beyond this range, special enhancements acting as localization limiters are necessary.

From the mathematical point of view, the onset of localization can be characterized as the appearance of a discontinuity in the strain rate, which later develops into a discontinuity in the strain itself. In one dimension, such a discontinuity can appear only if the tangent stiffness ceases to be positive. This means that localization is closely related to softening. In multiple dimensions, the relation between localization and softening is less straightforward. Localization may occur even in the hardening regime due to other destabilizing effects such as non-associated format of the evolution equations for internal variables (e.g. non-associated flow rule in plasticity). The localization conditions also depend on the specific stress state, and certain types of loading may delay the onset of localization far beyond the peak of the stress-strain diagram.

The purpose of this paper is to explore in detail the conditions that must be satisfied at the onset of localization for isotropic damage models with a single scalar damage variable. This is the basic class of damage models, which has well-known deficiencies but due to its simplicity is frequently used in numerical simulations. 


\section{Localization condition in general form}

The fundamental question addressed here is under which conditions the inelastic strain increments can localize in one or more narrow bands separated from the remaining part of the body by weak discontinuity surfaces. Across such surfaces, the displacement field remains continuous but the strain field can have a jump. At the onset of localization, the current strains are still continuous and the jump appears only in the strain rates. The necessary condition for the existence of such a solution has been established based on the classical localization analysis inspired by the early works of Hadamard [2] and Hill [3] and developed, among others, for plasticity in [8, 6] and for damage in [7]. The weak discontinuity can appear only if the determinant of the so-called localization tensor $Q$ becomes nonpositive. The localization tensor is defined as

$$
Q=n \cdot D \cdot n
$$

where $\boldsymbol{D}$ is the (tangent) stiffness tensor and $\boldsymbol{n}$ is a unit vector perpendicular to the discontinuity surface. If, for the material state at a given point, the determinant of $\boldsymbol{Q}$ is positive for all directions $\boldsymbol{n}$, all components of the strain rate must remain continuous at that point. The formation of a band with localized strain can thus begin only if there exists a certain direction $\boldsymbol{n}$ for which $\operatorname{det} \boldsymbol{Q} \leq 0$. If the tangent stiffness evolves continuously, this condition is first satisfied when

$$
\operatorname{det} \boldsymbol{Q}=0
$$

for some direction $\boldsymbol{n}$. From the mathematical point of view, singularity of the localization tensor indicates the so-called loss of ellipticity.

\section{Isotropic damage models}

The localization condition presented in the previous section is fairly general. For specific constitutive models, its particular form can be constructed by substituting the appropriate expression for the tangent stiffness tensor. In this paper, we will consider the class of isotropic damage models with one scalar damage variable $\omega$, described by the stress-strain law

$$
\boldsymbol{\sigma}=(1-\omega) \boldsymbol{D}_{e}: \boldsymbol{\varepsilon}
$$

damage law

$$
\omega=g(\kappa)
$$

and loading-unloading conditions

$$
f(\varepsilon, \kappa) \equiv \varepsilon_{\mathrm{eq}}(\varepsilon)-\kappa \leq 0, \quad \dot{\kappa} \geq 0, \quad f(\varepsilon, \kappa) \dot{\kappa}=0
$$

in which $f$ is the damage loading function, $g$ is the damage evolution function, $\varepsilon_{\text {eq }}$ is a scalar measure of the strain level called the equivalent strain, and $\kappa$ is an internal variable that corresponds to the maximum level of equivalent strain ever reached in the previous history of the material. The choice of the specific expression for the equivalent strain directly affects the shape of the elastic domain in the strain space and, as will be shown in Section, also the localization properties of the model.

From the rate form of the basic equations it is easy to derive the (elastic-damaged) tangent stiffness tensor

$$
\boldsymbol{D}_{e d}=(1-\omega) \boldsymbol{D}_{\mathrm{e}}-g^{\prime} \overline{\boldsymbol{\sigma}} \otimes \boldsymbol{\eta}
$$


Here, $\boldsymbol{D}_{\mathrm{u}}=(1-\omega) \boldsymbol{D}_{\mathrm{e}}$ is the unloading stiffness, $g^{\prime}=\mathrm{d} g / \mathrm{d} \kappa$ is the derivative of the damage function $g, \overline{\boldsymbol{\sigma}}=\boldsymbol{D}_{e}: \boldsymbol{\varepsilon}$ is the effective stress, and $\boldsymbol{\eta}=\partial \varepsilon_{\mathrm{eq}} / \partial \boldsymbol{\varepsilon}$ is a second order tensor obtained by differentiation of the expression for equivalent strain with respect to the strain tensor. The corresponding localization tensor is evaluated as

$$
\boldsymbol{Q}_{\mathrm{ed}}=(1-\omega) \boldsymbol{Q}_{\mathrm{e}}-g^{\prime}(\boldsymbol{n} \cdot \overline{\boldsymbol{\sigma}}) \otimes(\boldsymbol{\eta} \cdot \boldsymbol{n})=\boldsymbol{Q}_{\mathrm{u}}-g^{\prime} \overline{\boldsymbol{\sigma}}_{\mathrm{n}} \otimes \boldsymbol{\eta}_{\mathrm{n}}
$$

where $\overline{\boldsymbol{\sigma}}_{\mathrm{n}}=\boldsymbol{n} \cdot \overline{\boldsymbol{\sigma}}$ is the effective traction vector on the potential discontinuity plane, $\boldsymbol{\eta}_{\mathrm{n}}=\boldsymbol{\eta} \cdot \boldsymbol{n}$ is the projection of tensor $\boldsymbol{\eta}$ on that plane, and $\boldsymbol{Q}_{\mathrm{u}}=\boldsymbol{n} \cdot \boldsymbol{D}_{\mathrm{u}} \cdot \boldsymbol{n}=(1-\omega) \boldsymbol{n} \cdot \boldsymbol{D}_{\mathrm{e}} \cdot \boldsymbol{n}=(1-\omega) \boldsymbol{Q}_{\mathrm{e}}$ is a scalar multiple of the elastic acoustic tensor $\boldsymbol{Q}_{\mathrm{e}}=\boldsymbol{n} \cdot \boldsymbol{D}_{\mathrm{e}} \cdot \boldsymbol{n}=\boldsymbol{n} \cdot\left(\lambda \boldsymbol{\delta} \otimes \boldsymbol{\delta}+2 \mu \boldsymbol{I}_{s}\right) \cdot \boldsymbol{n}=(\lambda+\mu) \boldsymbol{n} \otimes \boldsymbol{n}+\mu \boldsymbol{\delta}$, in which $\lambda$ and $\mu$ are Lamé's coefficients. As shown for instance in [4], the localization tensor $Q_{\text {ed }}$ is singular if and only if

$$
g^{\prime} \boldsymbol{\eta}_{\mathrm{n}} \cdot \boldsymbol{Q}_{\mathrm{u}}^{-1} \cdot \overline{\boldsymbol{\sigma}}_{\mathrm{n}}=1
$$

The product $\boldsymbol{\eta}_{\mathrm{n}} \cdot \boldsymbol{Q}_{\mathrm{u}}^{-1} \cdot \overline{\boldsymbol{\sigma}}_{\mathrm{n}}$ depends on the elastic constants, on the current state of the material and on the assumed direction of discontinuity plane. For a given material state, it is continuous as a function of the unit vector $\boldsymbol{n}$ and has a maximum with respect to $\boldsymbol{n}$. Variable $g^{\prime}$ (the derivative of the damage function with respect to the equivalent strain) indicates how "dramatically" damage evolves. If $g^{\prime}$ is sufficiently small, condition (8) is not satisfied for any $\boldsymbol{n}$ and discontinuous bifurcations are excluded. The minimum value of $g^{\prime}$ that is needed for the loss of ellipticity is

$$
g_{\text {crit }}^{\prime}=\frac{1}{\max _{\|\boldsymbol{n}\|=1}\left(\boldsymbol{\eta}_{\mathrm{n}} \cdot \boldsymbol{Q}_{\mathrm{u}}^{-1} \cdot \overline{\boldsymbol{\sigma}}_{\mathrm{n}}\right)}
$$

If $g^{\prime}<g_{\text {crit }}^{\prime}$, the localization tensor $\boldsymbol{Q}_{\text {ed }}$ is regular for all possible directions $\boldsymbol{n}$, and a discontinuity in the strain rate is excluded. If $g^{\prime}=g_{c r i t}^{\prime}$, the localization tensor $\boldsymbol{Q}_{\text {ed }}$ is singular for that particular direction $\boldsymbol{n}$ which maximizes $\boldsymbol{\eta}_{\mathrm{n}} \cdot \boldsymbol{Q}_{\mathrm{u}}^{-1} \cdot \overline{\boldsymbol{\sigma}}_{\mathrm{n}}$, and a discontinuity across a plane perpendicular to that direction can start evolving. Finally, if $g^{\prime}>g_{\text {crit }}^{\prime}$, there exist infinitely many directions $\boldsymbol{n}$ for which $\boldsymbol{Q}_{\text {ed }}$ is singular, and the discontinuity can evolve even "more easily".

\section{Localization analysis in one dimension}

To get more insight into the meaning of the critical value of $g^{\prime}$, let us reduce the results derived so far to the simplest case of a one-dimensional damage model. All tensors become scalars, the elastic stiffness tensor $\boldsymbol{D}_{\mathrm{e}}$ is replaced by Young's modulus $E$, the equivalent strain $\varepsilon_{\text {eq }}$ is the strain $\varepsilon$ itself (we consider monotonic tensile loading), and tensor $\boldsymbol{\eta}$ is replaced by the scalar $\eta=\mathrm{d} \varepsilon_{\text {eq }} / \mathrm{d} \varepsilon=1$. The unit normal vector $\boldsymbol{n}$ is also replaced by the scalar $n=1$, and so there is no difference between the localization tensor and the tangent stiffness. Realizing that the effective stress is $\bar{\sigma}=E \varepsilon$ and substituting into (6) we get the tangent stiffness

$$
E_{\mathrm{ed}}=E_{\mathrm{u}}-g^{\prime} \bar{\sigma} \eta=(1-\omega) E-g^{\prime} E \varepsilon=E\left(1-\omega-g^{\prime} \varepsilon\right)
$$

The localization condition $\operatorname{det} \boldsymbol{Q}_{\text {ed }}=0$ is now written as $E_{\text {ed }}=0$, which means that a discontinuous bifurcation occurs when the peak of the stress-strain curve is reached. This is of course the result that we would expect intuitively. The value of $g^{\prime}$ corresponding to vanishing tangent stiffness $E_{\text {ed }}$ is

$$
g_{\text {crit }}^{\prime}=\frac{1-\omega}{\varepsilon}
$$

This is exactly what we obtain from the general formula (9) by substituting 1 for $\boldsymbol{\eta}_{\mathrm{n}},(1-\omega) E$ for $\boldsymbol{Q}_{\mathrm{u}}$ and $E \varepsilon$ for $\overline{\boldsymbol{\sigma}}_{\mathrm{n}}$. 


\section{Localization analysis in two dimensions (plane stress conditions)}

Evaluation of the critical value of $g^{\prime}$ was, in the one-dimensional case, very easy because no maximization with respect to $\boldsymbol{n}$ was needed. Let us now proceed to the analysis of a two-dimensional model under the assumptions of plane stress.

The elastic stiffness tensor for a two-dimensional plane-stress model is written as

$$
\boldsymbol{D}_{\mathrm{e}}=2 G\left(\boldsymbol{I}+\frac{\nu}{1-\nu} \boldsymbol{\delta} \otimes \boldsymbol{\delta}\right)
$$

where $G \equiv \mu$ is the elastic shear modulus. The corresponding elastic acoustic tensor

$$
\boldsymbol{Q}_{\mathrm{e}}=\boldsymbol{n} \cdot \boldsymbol{D}_{\mathrm{e}} \cdot \boldsymbol{n}=G\left(\boldsymbol{\delta}+\frac{1+\nu}{1-\nu} \boldsymbol{n} \otimes \boldsymbol{n}\right)
$$

can easily be inverted:

$$
\boldsymbol{Q}_{\mathrm{e}}^{-1}=\frac{1}{G}\left(\boldsymbol{\delta}-\frac{1+\nu}{2} \boldsymbol{n} \otimes \boldsymbol{n}\right)
$$

Note that all terms in the product $\boldsymbol{\eta}_{\mathrm{n}} \cdot \boldsymbol{Q}_{\mathrm{u}}^{-1} \cdot \overline{\boldsymbol{\sigma}}_{\mathrm{n}}$ depend on the unit normal vector $\boldsymbol{n}$. Vectors $\boldsymbol{\eta}_{\mathrm{n}}$ and $\overline{\boldsymbol{\sigma}}_{\mathrm{n}}$ are respectively defined as $\boldsymbol{\eta} \cdot \boldsymbol{n}$ and $\overline{\boldsymbol{\sigma}} \cdot \boldsymbol{n}$, where $\boldsymbol{\eta}$ and $\overline{\boldsymbol{\sigma}}$ are, for a given material state, fixed symmetric second-order tensors. Since the inverse of $\boldsymbol{Q}_{\mathrm{u}}$ is $\boldsymbol{Q}_{\mathrm{u}}^{-1}=\boldsymbol{Q}_{\mathrm{e}}^{-1} /(1-\omega)$, the function to be maximized with respect to $\boldsymbol{n}$ can be presented as

$$
f(\boldsymbol{n})=\boldsymbol{\eta}_{\mathrm{n}} \cdot \boldsymbol{Q}_{\mathrm{u}}^{-1} \cdot \overline{\boldsymbol{\sigma}}_{\mathrm{n}}=\frac{1}{(1-\omega) G}\left[\boldsymbol{n} \cdot \boldsymbol{\eta} \cdot \overline{\boldsymbol{\sigma}} \cdot \boldsymbol{n}-\frac{1+\nu}{2}(\boldsymbol{n} \cdot \boldsymbol{\eta} \cdot \boldsymbol{n})(\boldsymbol{n} \cdot \overline{\boldsymbol{\sigma}} \cdot \boldsymbol{n})\right]
$$

The principal directions of tensors $\overline{\boldsymbol{\sigma}}=\boldsymbol{D}_{\mathrm{e}}: \boldsymbol{\varepsilon}$ and $\boldsymbol{\eta}=\partial \varepsilon_{\mathrm{eq}} / \partial \varepsilon$ are the same, and if we rewrite the bracketed expression in (15) in terms of the components with respect to the principal coordinate system, we obtain the fourth-order polynomial

$$
f^{*}\left(n_{1}, n_{2}\right)=\eta_{1} \bar{\sigma}_{1} n_{1}^{2}+\eta_{2} \bar{\sigma}_{2} n_{2}^{2}-\frac{1+\nu}{2}\left(\eta_{1} n_{1}^{2}+\eta_{2} n_{2}^{2}\right)\left(\bar{\sigma}_{1} n_{1}^{2}+\bar{\sigma}_{2} n_{2}^{2}\right)
$$

This polynomial has to be maximized, subject to the normalizing constraint $n_{1}^{2}+n_{2}^{2}=1$. Renaming $n_{1}^{2}$ as $N_{1}$ and $n_{2}^{2}$ as $N_{2}=1-N_{1}$, we convert the objective function $f$ into a quadratic function of one single argument $N_{1}$ and we automatically satisfy the normalizing constraint, but additional inequality constraints $0 \leq N_{1} \leq 1$ must be imposed. The coefficient multiplying the quadratic term $N_{1}^{2}$ is $-\left(\eta_{1}-\eta_{2}\right)\left(\bar{\sigma}_{1}-\bar{\sigma}_{2}\right)$. Without any loss of generality, we can order the principal stresses such that $\bar{\sigma}_{1} \geq \bar{\sigma}_{2}$. The equality sign corresponds to the special cases of equibiaxial tension or equibiaxial compression. In all other cases, the function to be maximized is strictly concave, provided that $\eta_{1}>\eta_{2}$ (which is usually verified for the commonly used expressions for equivalent strain). The value of $N_{1}$ at which the first derivative of $f^{*}$ vanishes is

$$
N_{1}^{*}=\frac{\eta_{1} \bar{\sigma}_{1}+\nu \eta_{2} \bar{\sigma}_{2}-(1+\nu)\left(\eta_{1} \bar{\sigma}_{2}+\eta_{2} \bar{\sigma}_{1}\right) / 2}{(1+\nu)\left(\eta_{1}-\eta_{2}\right)\left(\bar{\sigma}_{1}-\bar{\sigma}_{2}\right)}
$$

If $N_{1}^{*}$ is between 0 and 1 , the unit normal to the potential discontinuity curve has coordinates $n_{1, \text { crit }}=$ $\pm \sqrt{N_{1}^{*}}$ and $n_{2, \text { crit }}= \pm \sqrt{1-N_{1}^{*}}$. Formally, four solutions are obtained, but only two of them represent physically different directions, which are symmetrically placed with respect to the principal axes. If formula (17) gives $N_{1}^{*} \geq 1$, then the unit normal has components $n_{1, \text { crit }}=1$ and $n_{2, \text { crit }}=0$ and the discontinuity is perpendicular to the major principal axis. If formula (17) gives $N_{1}^{*} \leq 0$, then the unit normal has components $n_{1, \text { crit }}=0$ and $n_{2 \text {,crit }}=1$ and the discontinuity is perpendicular to the minor principal axis. 
In all the cases, the critical value of $g^{\prime}$ according to (9) is given by $g_{\text {crit }}^{\prime}=(1-\omega) G / f^{*}\left(n_{1, \text { crit }}, n_{2, \text { crit }}\right)$. Instead of looking at $g_{\text {crit }}^{\prime}$, it is more instructive to assess the corresponding critical value of tangent modulus $E_{\text {ed }}$, given by

$$
E_{\text {ed,crit }}=E\left(1-\omega-g_{\text {crit }}^{\prime} \varepsilon_{\text {eq }}\right)=E\left[1-\omega-\frac{(1-\omega) G \varepsilon_{\text {eq }}}{f^{*}\left(n_{1, \text { crit }}, n_{2, \text { crit }}\right)}\right]=E_{\mathrm{u}}\left[1-\frac{G \varepsilon_{\text {eq }}}{f^{*}\left(n_{1, \text { crit }}, n_{2, \text { crit }}\right)}\right]
$$

In general, the orientation of the potential discontinuity and the critical ratio between the tangent and unloading moduli depend on the current state, which enters through the principal values of $\boldsymbol{\eta}$ and $\bar{\sigma}$; see (16)-(17). To illustrate the influence of the particular definition of equivalent strain on the localization properties of the model, let us consider the following choices:

- Mazars definition of equivalent strain [5]:

$$
\begin{aligned}
\varepsilon_{\mathrm{eq}} & =\|\langle\varepsilon\rangle\|=\sqrt{\sum_{I=1}^{3}\left\langle\varepsilon_{I}\right\rangle^{2}} \\
\boldsymbol{\eta} & =\frac{\partial \varepsilon_{\mathrm{eq}}}{\partial \boldsymbol{\varepsilon}}=\frac{\langle\varepsilon\rangle}{\varepsilon_{\mathrm{eq}}}
\end{aligned}
$$

where $\varepsilon_{I}, I=1,2,3$, are the principal strains, and the brackets $\langle\ldots\rangle$ denote the positive part.

- Rankine-type definition of equivalent strain:

$$
\begin{aligned}
\varepsilon_{\mathrm{eq}} & =\frac{\bar{\sigma}_{1}}{E} \\
\boldsymbol{\eta} & =\frac{\partial \varepsilon_{\mathrm{eq}}}{\partial \boldsymbol{\varepsilon}}=\frac{1}{E} \frac{\partial \bar{\sigma}_{1}}{\partial \overline{\boldsymbol{\sigma}}}: \frac{\partial \overline{\boldsymbol{\sigma}}}{\partial \boldsymbol{\varepsilon}}=\frac{1}{E}\left(\boldsymbol{p}_{1} \otimes \boldsymbol{p}_{1}\right): \boldsymbol{D}_{e}=\frac{1}{1+\nu}\left(\frac{\nu}{1-2 \nu} \boldsymbol{\delta}+\boldsymbol{p}_{1} \otimes \boldsymbol{p}_{1}\right)
\end{aligned}
$$

where $\bar{\sigma}_{1}$ is the maximum principal effective stress and $\boldsymbol{p}_{1}$ is the unit vector in the corresponding principal direction.

- Modified von Mises definition of equivalent strain [1]:

$$
\varepsilon_{\mathrm{eq}}=\frac{(k-1) I_{1 \varepsilon}}{2 k(1-2 \nu)}+\frac{1}{2 k} \sqrt{\frac{(k-1)^{2}}{(1-2 \nu)^{2}} I_{1 \varepsilon}^{2}+\frac{12 k J_{2 \varepsilon}}{(1+\nu)^{2}}}
$$

where

$$
I_{1 \varepsilon}=\boldsymbol{\delta}: \varepsilon
$$

is the first strain invariant (trace of the strain tensor),

$$
J_{2 \varepsilon}=\frac{1}{2} \boldsymbol{e}: \boldsymbol{e}=\frac{1}{2} \varepsilon: \boldsymbol{\varepsilon}-\frac{1}{6} I_{1 \varepsilon}^{2}
$$

is the second deviatoric strain invariant, and $k$ is a model parameter controling the ratio between the uniaxial compressive strength $f_{\mathrm{c}}$ and uniaxial tensile strength $f_{\mathrm{t}}$. 
(a)

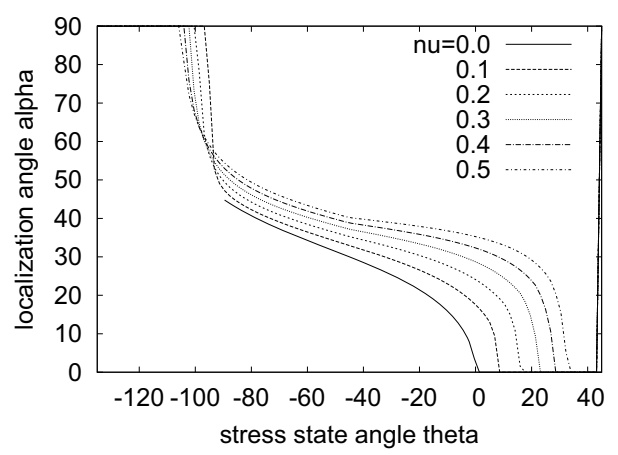

(b)

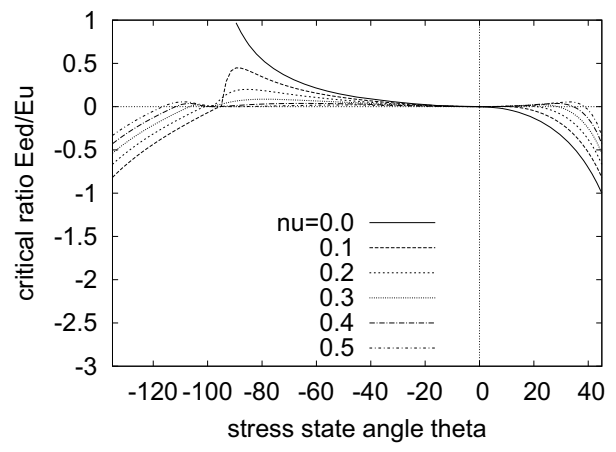

Figure 1: Localization characteristics of model with Mazars equivalent strain: (a) dependence of localization angle on stress state, (b) dependence of critical tangent modulus on stress state.

All the above expressions have been written for the full three-dimensional formulation. In planestress analysis, we work only with the in-plane components of stress and strain, but the fact that the out-of-plane normal strain $\varepsilon_{33}$ is nonzero is taken into account. For isotropic elastic materials, the plane stress condition $\sigma_{33}=0$ leads to $\varepsilon_{33}=-\left(\varepsilon_{11}+\varepsilon_{22}\right) \nu /(1-\nu)$, and this relation remains valid for the present class of one-parameter isotropic damage models even after the onset of damage. The equivalent strain is rewritten in terms of the in-plane components only and its derivatives are evaluated according to the chain rule. For instance, for the Mazars definition, the values of $\eta_{1}$ and $\eta_{2}$ to be substituted into (16)-(17) are

$$
\begin{aligned}
& \eta_{1}=\frac{\partial \varepsilon_{\mathrm{eq}}}{\partial \varepsilon_{1}}+\frac{\partial \varepsilon_{\mathrm{eq}}}{\partial \varepsilon_{3}} \frac{\partial \varepsilon_{3}}{\partial \varepsilon_{1}}=\frac{\left\langle\varepsilon_{1}\right\rangle}{\varepsilon_{\mathrm{eq}}}+\frac{\left\langle\varepsilon_{3}\right\rangle}{\varepsilon_{\mathrm{eq}}}\left(-\frac{\nu}{1-\nu}\right)=\frac{1}{\varepsilon_{\mathrm{eq}}}\left(\left\langle\varepsilon_{1}\right\rangle-\frac{\nu^{2}}{(1-\nu)^{2}}\left\langle-\varepsilon_{1}-\varepsilon_{2}\right\rangle\right) \\
& \eta_{2}=\frac{1}{\varepsilon_{\mathrm{eq}}}\left(\left\langle\varepsilon_{2}\right\rangle-\frac{\nu^{2}}{(1-\nu)^{2}}\left\langle-\varepsilon_{1}-\varepsilon_{2}\right\rangle\right)
\end{aligned}
$$

The results of plane-stress localization analysis of the isotropic damage model with damage driven by equivalent strain given by one of the above definitions are graphically presented in Figs. 1-3. The direction of potential discontinuity is described by the localization angle $\alpha$, which is the angle between the normal to the discontinuity and the major principal axis of strain and stress. The cosine and sine of the localization angle are the components $n_{1, \text { crit }}$ and $n_{2, \text { crit }}$ of the unit normal vector. This means that $\alpha$ is evaluated as $\alpha=\arccos n_{1, \text { crit }}=\arccos \sqrt{N_{1}^{*}}$. The critical "rate" of damage growth needed for localization is described indirectly by the critical ratio between the tangent and unloading moduli, $E_{\text {ed,crit }} / E_{\mathrm{u}}$, evaluated from (18). Both characteristics depend on the specific choice of the expression for equivalent strain, on the stress state and Poisson's ratio. The stress state is sufficiently characterized by the ratio of the principal in-plane stresses, $\bar{\sigma}_{2} / \bar{\sigma}_{1}$. It is convenient to introduce an angle $\theta$ such that $\bar{\sigma}_{1}=c \cos \theta$ and $\bar{\sigma}_{2}=c \sin \theta$ where $c \geq 0$ is an undetermined multiplier. For $\bar{\sigma}_{1} \geq \bar{\sigma}_{2}, \theta$ varies between $-135^{\circ}$ for equibiaxial compression and $45^{\circ}$ for equibiaxial tension. The values $\theta=-90^{\circ},-45^{\circ}$ and $0^{\circ}$ respectively correspond to uniaxial compression, shear and uniaxial tension.

The results of localization analysis are somewhat surprizing. Consider first the Mazars definition of equivalent strain. According to Fig. 1a, the localization angle $\alpha$ is equal to $0^{\circ}$ only for stress states close to equibiaxial tension. Under uniaxial tension, it is $0^{\circ}$ only if the Poisson ratio vanishes. For typical values of Poisson ratio around 0.2 , the discontinuity tends to form in an inclined direction, not 
(a)

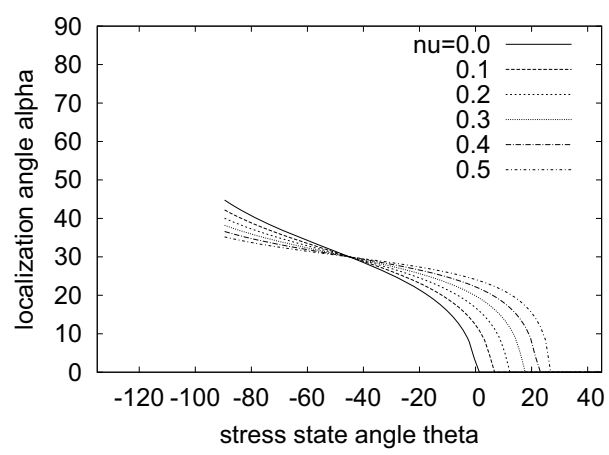

(b)

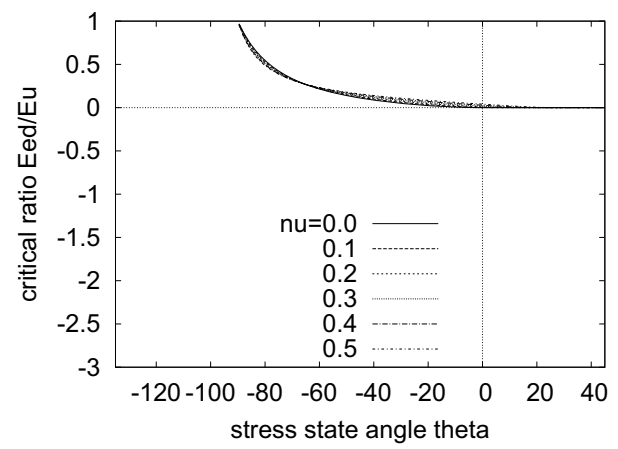

Figure 2: Localization characteristics of model with Rankine equivalent strain: (a) dependence of localization angle on stress state, (b) dependence of critical tangent modulus on stress state.

(a)

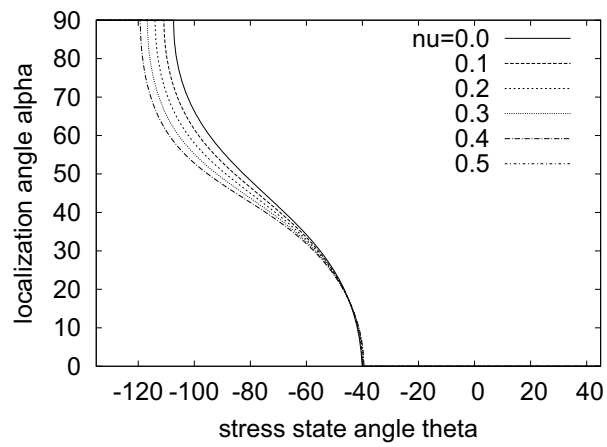

(b)

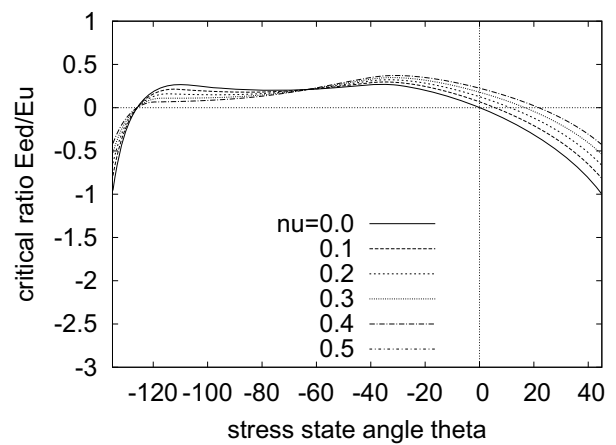

Figure 3: Localization characteristics of model with modified Mises equivalent strain: (a) dependence of localization angle on stress state, (b) dependence of critical tangent modulus on stress state.

perpendicular to the direction of loading. Under combined tension and compression, the localization angle increases and for uniaxial compression it is above $45^{\circ}$. The critical value of tangent modulus, plotted in Fig. 1b, is positive in the entire range of stress states combining tension in one direction with lateral compression in the perpendicular direction. So for this type of stress states, localization can occur even before the peak of the stress-strain diagram. For uniaxial tension, the critical tangent modulus is exactly zero for all values of Poisson ratio, and localization can be expected right at peak. For biaxial tension, the critical modulus is positive for states with a large difference between principal stresses and negative for states close to equibiaxial tension.

Next, let us discuss the results for the Rankine definition of equivalent strain (Fig. 2). For states with both principal stresses nonpositive, the equivalent strain is zero and the damage threshold is never reached, so the model response remains elastic and localization cannot occur. For combined tensile-compressive stress states, the localization angle is roughly between $20^{\circ}$ and $40^{\circ}$, and the corresponding critical modulus is positive. So once again, localization can occur already in the hardening regime. For uniaxial and biaxial tension, the critical modulus is only slightly positive, and the localization angle becomes $0^{\circ}$ for states with a certain minimum level of lateral tension. 
Finally, consider the modified Mises definition of equivalent strain (Fig. 3). For uniaxial tension, the localization angle is zero, and so the discontinuity would be perpendicular to the loading direction. The same holds for biaxial tension and for tension combined with limited lateral compression. The critical modulus is positive and quite large for almost all stress states with the exception of those close to equibiaxial tension or equibiaxial compression. So the localization may occur way before the peak of the stress-strain diagram.

\section{Localization simulated by finite elements}

Some of the results of the theoretical localization analysis have been verified and illustrated by finite element simulations of a bar under uniaxial tension, discretized by two-dimensional finite elements (bilinear quads under plane stress). Skewed meshes containing layers of elements of different inclinations have been used, in order to allow for the formation of a localized damage band in an "arbitrary" direction. It turned out that, on such meshes, no imperfection was needed to trigger the bifurcation from a uniform state. The numerical solution spontaneously localized into one layer of elements with the most favorable orientation. This orientation was always very close to the theoretically predicted one. Due to space limitations, the corresponding figures are omitted here and they will be presented on the conference poster.

\section{Acknowledgment}

Financial support of the Czech Science Foundation under Project GAČR 106/08/1508 is gratefully acknowledged.

\section{References}

[1] J. H. P. de Vree, W. A. M. Brekelmans, and M. A. J. van Gils. Comparison of nonlocal approaches in continuum damage mechanics. Computers and Structures, 55:581-588, 1995.

[2] J. Hadamard. Leçons sur la propagation des ondes. Librairie Scientifique A. Hermann et Fils, Paris, 1903.

[3] R. Hill. A general theory of uniqueness and stability in elastic-plastic solids. Journal of the Mechanics and Physics of Solids, 6:236-249, 1958.

[4] M. Jirásek. Mathematical analysis of strain localization. Revue Française de Génie Civil, 11:997$1011,2007$.

[5] J. Mazars. Application de la mécanique de l'endommagement au comportement non linéaire et à la rupture du béton de structure. Thèse de Doctorat d'Etat, Université Paris VI., France, 1984.

[6] N. Ottosen and K. Runesson. Properties of discontinuous bifurcation solutions in elasto-plasticity. International Journal of Solids and Structures, 27:401-421, 1991.

[7] E. Rizzi, I. Carol, and K. Willam. Localization analysis of elastic degradation with application to scalar damage. Journal of Engineering Mechanics, ASCE, 121:541-554, 1996.

[8] J. W. Rudnicky and J. R. Rice. Conditions for the localization of deformation in pressure-sensitive dilatant materials. Journal of the Mechanics and Physics of Solids, 23:371-394, 1975. 